\title{
Rectal area as surrogate measure of rectal emptying during MR defecography
}

Patients with chronic constipation frequently report symptoms of difficult defecation such as excessive straining, feeling of incomplete evacuation and digital facilitation of bowel movements. Current functional investigations have not yet found specific alterations that explain these symptoms, perhaps in relation to the multi-dimensional pathophysiology of chronic constipation and to the strong influence that the voluntary control of the external anal sphincter might have on colorectal function. In the balloon expulsion test, rectal expulsion is evaluated by asking patients to expel balloons filled with water or air from the rectum. The normal time required to expel the balloon depends on the method used and lasts up to 1-2 minutes. A prolonged expulsion time has been found to predict the response to biofeedback treatment, but a normal test does not exclude a defecation disorder. Anorectal manometry includes measurement of anal sphincter pressures at rest and during voluntary contractions, anal sphincter relation and rectal sensitivity in response to rectal distension and measurement of anal pressures during attempted defecation. Studies with high resolution anorectal manometry have recently demonstrated that the "abnormal" pressure changes during attempted defecation, believed to underlie a difficult defecation, are unspecific as are often found in healthy subjects. Defecography is a radiologic or MRI based technique used to evaluate the rectum and pelvic floor morphology after administering barium or ultrasound gel in the rectum and during attempted defecation. This test can detect both structural (rectocele, enterocele, intussusception, rectal prolapse, and megarectum) and functional (not relaxing puborectalis during straining, perineal descent, inadequate rectal emptying after evacuation) abnormalities believed to underlie a difficult defecation. Magnetic resonance defecography has the advantages to include better resolution of soft tissues surrounding the rectum, to improve the ability to visualize anal sphincter and levator ani muscles, and the lack of radiation. The technique is not standardised across different centres and both structural and functional abnormalities found at defecography are often found also in healthy subjects, indicating that the role of defecography in the evidence based management of patients with difficult defecation remains unclear.

The present study from Puthanmadhom et al presents a software-based analysis of MRI defecography, that provides a more objective assessment of rectal area and volume after rectal administration of ultrasound gel and after evacuation in healthy subjects and constipated patients. The results are compared with those of the balloon expulsion test. Indeed, even if a semiautomated program for quantifying rectal emptying is available, barium defecography is generally performed by visually estimating the change in rectal area in the lateral view before and after evacuation, assuming that rectal area represents a surrogate measure of rectal volume during evacuation.

Previous studies with barium defecography reported an $82 \%$ reduction in rectal area after defecation in healthy subjects (Bartram 1988) with a wide range of variation (Palit 2013). The present data show that the percentage reduction in rectal volume after evacuation was lower in participants with an abnormal BET as compared to healthy subjects?? (maybe better to put some data here to give an idea of values), suggesting that the behavioural or visceral factors determining an abnormal BET impair the efficiency of evacuation. Whether the patients with abnormal BET and inefficient evacuation at MRI represent a specific subset of constipated patients amenable of specific treatment remains to be elucidated. Meanwhile the not negligible percentage of healthy subjects with an abnormal BET (maybe better to add also here the data) reminds that these alterations are not specific and that both BET and the MRI assessment of the efficiency of evacuation are strongly influenced by the attitude/embarrassment to accomplish the act of defecation in the laboratory and in response to an artificial stimulation (do you mean the lying position? If so I would mention this).

"Obstructed defecation" is one of the diagnosis often used to identify constipated patients with difficult defecation. The definition of obstruction in gastroenterology usually requires the dilation of the segment of the gut cranial to the obstruction. The present data shows that the volume of the 
empty? rectum of participants with abnormal BET was even smaller than the rectum of participants with normal BET indicating that the mechanisms underlying an abnormal BET do not cause a significant mechanical obstruction. Thus, the term of "obstructed defecation" should not be used to identify constipated patients with difficult defecation.

The changes in rectal area were strongly correlated to the changes in rectal volume and equivalent for distinguishing between normal or abnormal BET. The Authors concluded that changes in rectal area represent a reasonably accurate surrogate measure of rectal emptying during MR defecography. However, the differences between the two measures were greater in participants with a less efficient rectal evacuation at MRI or BET?, in which the proportion of patients with a rectocele $>2 \mathrm{~cm}$ was also greater, revealing that, at least in certain anatomical conditions, the assessment of rectal area might not be representative of rectal volume. As the Defecography defecography is a poerly standardized technique-often analysed with qualitative methods (Grossi) ifn this context, the present study prompts the application of software-based techniques to allow a quick automatic calculation of rectal areas and hopefully rectal volumes in order to provide a precise definition of investigated variables. New research should also consider that dedicated software might automatically draw the region of interest used to calculate the areas of each sagittal image, further minimizing the qualitative part of the analytical process.

The results of the study by Puthanmadhom et al (1) are not limited to the interpretation of MRI defecography, but they may give some clues on rectal physiology and on the interpretation of previous studies assessing rectal motor and sensory responses to distension. Anatomical textbook reports that the rectum is about $15 \mathrm{~cm}$ long and $4 \mathrm{~cm}$ large; assuming a cylindrical shape the calculated volume should be $188 \mathrm{ml}$. The results of the present study show that the capacity of the rectum after retrograde load of $180 \mathrm{ml}$ ultrasound gel was $65 \%$ of the administered volume (118 $\mathrm{ml}$ ), suggesting that this volume might represent the "normal" maximal volume of the rectum after retrograde load. Conflicting results were previously reported on rectal motor response to distension in term of rectal tone and compliance in healthy subjects and patients with irritable bowel syndrome (IBS). Some Authors (Kvan, Corsetti, Tornblom) suggested that these characteristics should have been investigated within "physiological" distension ranges in order to maximize the effects related to the active contractile properties of the rectal muscle and not at greater painful levels of distensions, when not compliant passive tissue components were more involved (Dal Lago). These "physiological" distension ranges remained poorly defined, but in fact a significant difference in rectal motor response to distensiontene was found when the volumes of rectal distension (within a 8 $\mathrm{cm}$ barostat bag inflated at the minimal distending pressure) were lower $(99 \mathrm{ml}$ in healthy subjects and $84 \mathrm{ml}$ in IBS) than the normal maximal volume of the present study (worth repeating these values here) $(99 \mathrm{ml}$ in healthy subjects and $84 \mathrm{ml}$ in $\mathrm{IBS})$. In line with these results the rectum of patients with IBS turned out to be iperreactive (Corsetti) and poorly compliant (Kvan e Tornblom) in response to relatively low volumes of distension.

After evacuation, the volume of the rectum become very small $(13 \mathrm{ml})$ in subjects with normal BET but it was significantly greater in subjects with abnormal BET $(25 \mathrm{ml})$ suggesting an abnormal retention after evacuation in the latter group. However even this volume was smaller than the volume of $44 \mathrm{ml}$ measured with MRI in resting conditions and without retrograde load in healthy subjects (Di Palma 2011) suggesting that the volume of an "empty" rectum might vary according to different experimental conditions.

In conclusion the study by Puthanmadhom et al should be greatly appreciated as it expands our understanding on rectal physiology, it provides some interesting clues on the interpretation of physiological tests performed in patients with functional bowel disorders and it finally prompts both radiologist and gastroenterologist to adopt software based systems of quantitative analysis of rectal volumes in order to explore with more robust data this complicated area of gastrointestinal function. 
In this prospective it is highly advocated that radiologists, gastroenterologists and MRI experts parallel the clinical success of cine cardiac MRI (see enclosed paper) and work together to improve and standardise MRI defecography. This will allow multicentre high quality studies to evaluate the role of anatomical and functional rectal alterations in constipated patients with difficult defecation. 